\title{
Hydrogen Bonding and Stereospecific Long-range Spin Coupling in 3-Nitro- and 5-Nitrosalicylaldehyde
}

\author{
STURE FORSÉN and B J ÖRN ÅKERMARK
}

Research Group for Nuclear Magnetic Resonance, Division of Physical Chemistry and Division of Organic Chemistry, The Royal Institute of Technology, Stockholm 70, Sweden

\begin{abstract}
The stereospecific nature of the long-range spin coupling between aldehydic protons and ring protons in aromatic aldehydes is used for determining the direction of the intramolecular hydrogen bond in 3-nitrosalicylaldehyde.

Indications are obtained that the long-range spin coupling of phenolic hydroxyl protons also is stereospecific.
\end{abstract}

The intramolecular hydrogen bond in 3-nitrosalicylaldehyde has recently 1 been the subject of several investigations with infrared spectroscopy ${ }^{1,2}$.<smiles>O=Cc1cccc([N+](=O)[O-])c1O</smiles>

I a<smiles>O=Cc1cccc2c1OCON2O</smiles>

I b

The spectral properties of the aldehyde group in 3-nitrosalicylaldehyde does not fit in the general pattern followed by other investigated salicylaldehyde derivatives and it has been suggested that the intramolecular hydrogen bond of 3-nitrosalicylaldehyde in inert solvents should be predominantly directed towards the nitro group ( $\mathrm{I}$ b) rather than to the aldehydic oxygen (I a).

A unique possibility of distinguishing between the two hydrogen bonded structures I a and I b has been offered through the recently demonstrated stereospecific long-range spin coupling of the aldehydic proton to the ring protons in aromatic aldehydes ${ }^{3-5}$. The stereospecific long-range spin coupling in benzaldehyde derivatives has the following distinctive peculiarities:

In 2-hydroxy substituted benzaldehydes where the aldehyde group is "locked" in the following configuration 
<smiles>COc1ccccc1C=O</smiles>

the aldehydic hydrogen has been found to be spin coupled to the ring hydrogen in the 3-position whereas no spin coupling has been observed to the other ring protons. In 2-substituted benzaldehydes where no appreciable specific attractive interaction between the 2 -substituent $(\mathrm{X})$ and the carbonylic oxygen is expected, the configuration with the carbonyl group turned away from the substituent,

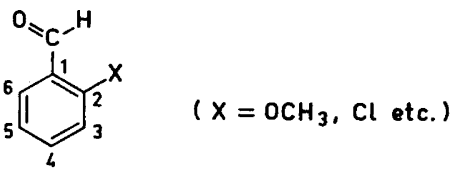

should be favoured on sterical grounds. In benzaldehyde derivatives of this type the aldehydic proton has been found to be spin coupled solely to the ring hydrogen in the 5-position.

The implication of this stereospecific long-range spin coupling of the aldehydic proton, in the case of 3-nitrosalicylaldehyde, is that if the phenolic $\mathrm{OH}$-group is hydrogen-bonded to the aldehydic oxygen (I a), one would expect no spin-spin splitting of the aldehydic proton as the 3-position is substituted. However, if the hydrogen bond is formed between the phenolic OH-group and the nitro group, one should expect the aldehyde proton to be spin coupled with the proton in the 5-position.

In 2-hydroxy substituted benzaldehydes, long-range spin coupling has also been observed between the phenolic hydroxyl proton and the ring proton in the 4-position ${ }^{\mathbf{3}, 4}$. The stereospecificity of this latter long-range spin coupling is not yet well established. However, if the long-range spin coupling of the hydroxyl proton is stereospecific, in analogy with the corresponding aldehydic spin coupling, one would expect the hydrogen bonded proton in structure I b to be spin coupled with the hydrogen in the 6-position.

In the present investigation the proton magnetic resonance (PMR) spectra of 3-nitrosalicylaldehyde and also of 5-nitrosalicylaldehyde have been obtained on solutions in deuteriochloroform. The implications of the observed spin coupling pattern for the direction of the intramolecular hydrogen bond in 3-nitrosalicylaldehyde is discussed.

\section{EXPERIMENTAL}

Methods. The PMR spectra were obtained with a Varian A-60 spectrometer at a temperature of $33.5^{\circ} \mathrm{C}$. The spectra recorded were of dilute solutions $(c a .5 \%)$ in deuteriochloroform containing $0.5 \%$ tetramethylsilane as internal reference. The chemical shifts $(\delta)$ have been calculated as $f_{\mathrm{i}}$-values ${ }^{6}$ and are given in ppm relative to tetramethylsilane.

The IR spectra were recorded with a Perkin-Elmer No. 21 spectrophotometer. Melting points were determined on a Kofler block.

Acta Chem. Scand. 17 (1963) No. 6 


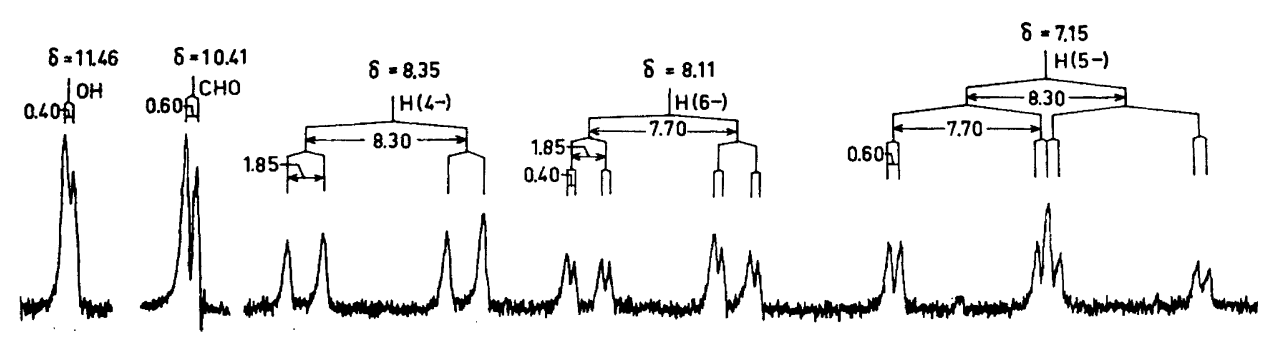

Fig. 1. The proton magnetic resonance spectrum of 3-nitrosalicylaldehyde in deuteriochloroform. The chemical shifts are given in ppm relative to an internal tetramethylsilane standard and the spin-spin splittings are in $\mathrm{c} / \mathrm{s}$. The aldehydic proton is spin coupled to the ring proton in the 5-position and the phenolic proton to the ring hydrogen in the 6-position.

Materials. 2-Hydroxy-3-nitrobenzaldehyde and 2-hydroxy-5-nitrobenzaldehyde. Salicylaldehyde was nitrated according to Miller ${ }^{7}$ and 2-hydroxy-5-nitrobenzaldehyde, m.p. $127^{\circ}$ (sublimed), isolated as the sodium salt ${ }^{7}$. The mother liquors were acidified and the precipitate $(1.4 \mathrm{~g})$ chromatographed on silica gel using light petroleum $\left(40^{\circ}-60^{\circ}\right)$ in benzene $(50-0 \%)$ and ether in benzene $(0-50 \%)$ as developers. The middle fractions gave a small amount pure 2-hydroxy-3-nitrobenzaldehyde $(0.11 \mathrm{~g})$, m.p. $106^{\circ}-107^{\circ}$ (sublimed), free from the 5-nitro-isomer according to PMR and IR mesurements.

\section{RESULTS AND DISCUSSION}

The PMR spectrum of 3-nitrosalicylaldehyde is presented in Fig. 1. Whereas the assignment of the multiplet arising from the hydrogen in the 5-position is evident from the splitting due to spin-spin coupling, the assignment of the signals from the hydrogens in the 4- and 6-positions may not be deduced in this simple way. However, the chemical shifts of the ring protons in substituted benzenes show a considerable regularity with the nature of the substituent 8,9 . Hydrogens ortho to nitro groups are generally observed to be shifted more towards lower fields than are hydrogens ortho to aldehyde groups, and in the case of 3-nitrosalicylaldehyde this leads to the assignment shown in Fig. 1.

Both the signal from the aldehyde group and the signal from the hydroxyl proton are found to be doublets, due to spin coupling to ring protons. The splitting of the aldehyde signal is clearly due to long-range spin coupling with the hydrogen in the 5-position. In view of the stereospecificity of the aldehydic long-range coupling discussed above, this result strongly indicates that the hydrogen bond in 3-nitrosalicylaldehyde is predominantly directed towards the nitro group $(\mathrm{I} \mathrm{b})$. The observed splitting $(0.60 \pm 0.03 \mathrm{c} / \mathrm{s})$ of the aldehyde signal should, to within a few percent, equal the spin coupling constant, $J_{\mathrm{CHO}-\mathrm{H}_{(5-)}}$, since the 5-hydrogen is not strongly coupled to the other ring protons.

The splitting of the signal from the $\mathrm{OH}$-group is clearly due to spin coupling with the ring hydrogen in the 6-position and this is an indication that the longrange spin coupling of phenolic hydroxyl protons is also stereospecific. A larger body of spectral evidence is, however, certainly necessary before the stereospecificity can be regarded as well established. 

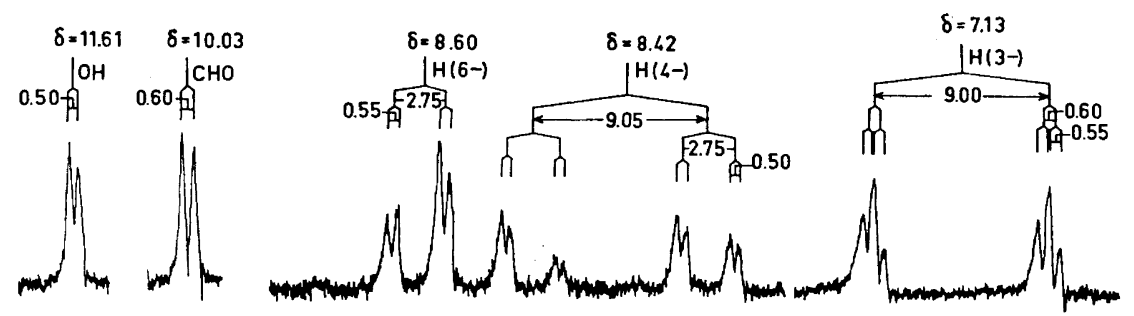

Fig. 2. The proton magnetic resonance spectrum of 5-nitrosalicylaldehyde in deuteriochloroform. The chemical shifts are given in ppm relative to an internal tetramethylsilane standard and the spin-spin splittings are in c/s. The aldehydic proton is spin coupled to the ring lproton in the 3-position and phenolic proton to the ring hydrogen in the 4-position (see text).

It was observed that the spin coupling of the hydroxyl proton with the 6-hydrogen could be removed, without any noticeable effect on the longrange spin coupling of the aldehyde proton, by means of a small amount of triethylamine added to the deuteriochloroform solutions. A similar effect has also been observed for the spin coupling of the hydroxyl proton in a number of salicylaldehyde derivatives and has proved of great value in simplifying the PMR spectra of the ring protons and facilitating assignment of the signals.

This may be exemplified in the case of 5-nitrosalicylaldehyde. The PMR spectrum of this compound in deuteriochloroform is shown in Fig. 2. The assignment of the signal groups to the respective ring hydrogens is obtained from a consideration of the spin coupling pattern. The signals from the hydroxyl and aldehyde protons are doublets, due to spin coupling with the ring hydrogens. Since the splittings of the $\mathrm{OH}$ and $\mathrm{CHO}$ signals, as well as the smallest splitting in the signal groups from the ring hydrogens are nearly equal $(0.55 \pm 0.05 \mathrm{c} / \mathrm{s})$, it may not be entirely obvious to which ring hydrogens the hydroxylic and the aldehyde protons are spin coupled. Upon addition of a small amount of triethylamine the $\mathrm{OH}$ signal is considerably broadened and the small splitting in the signal group due to $H(4-)$ disappears, whereas the splitting of the signals due to the aldehyde group and to the ring hydrogens $\mathrm{H}(3-)$ and $\mathrm{H}(6-)$ remains almost unchanged. This confirms that the splitting of the hydroxyl proton in Fig. 2 is due to spin coupling with the hydrogen $\mathrm{H}(4-)$ and also confirms the anticipated long-range spin coupling between the aldehyde proton and the ring hydrogen $\mathrm{H}(3-)$.

The resonance position of the aldehyde proton in 3-nitrosalicylaldehyde is shifted $0.4 \mathrm{ppm}$ towards lower field compared with the resonance position in 5-nitrosalicylaldehyde. This seems to further confirm the hydrogen bonded structure I b for 3-nitrosalicylaldehyde since the molecular environment of the aldehydic proton in $I \mathrm{~b}$ should be quite different from that in structure $\mathrm{I} a$ and in 5-nitrosalicylaldehyde, in which the aldehyde group is "locked" by the intramolecular hydrogen bond with the aldehydic proton pointing away from the neighbouring substituents. Both diamagnetic anisotropy effects due to the phenolic $\mathrm{C}-\mathrm{O}$ bond and electric distortion effects produced by the lone-pair dipoles of the phenolic oxygen could contribute to the low field shift of the aldehyde proton in structure $I \mathrm{~b}$. 
Acknowledgements. The authors like to thank Professor H. Erdtman, Dr. E. Forslind and Professor O. Lamm for facilities put at our disposal and Dr. E. Forslind also for valuable discussions. The work has been sponsored by a grant from the Swedish Technical Research Council and the cost of the NMR spectrometer has been defrayed by a grant from Knut and Alice Wallenbergs Stiftelse.

\section{REFERENCES}

1. Hoyer, H. and Hensel, W. Z. Elektrochem. 64 (1960) 958.

2. Brocks, C. J. W. and Morman, J. F. J. Chem. Soc. 19613372.

3. For a recent discussion of long-range couplings between proton spins see paper by C. N. Banwell and N. Sheppard, presented at the General Discussion on High Resolution NMR, arranged by the Faraday, Soc., Oxford Sept. 1962. The paper will appear in Discussions Faraday Soc. 1963.

4. Forsén, S. and Åkermark, B. To be published.

5. de Kowalewski, D. G. and Kowalewski, W. J. J. Chem. Phys. 36 (1962) 266.

6. Hoffman, R. A. and Gronowitz, S. Arkiv Kemi 16 (1960) 515.

7. v. Miller, W. Ber. 20 (1887) 1927.

8. Diehl, P. Helv. Chim. Acta 44 (1961) 829.

9. Martin, J. and Dailey, B. P. J. Chem. Phys. 37 (1962) 2594.

Received April 5, 1963. 